\section{Differences in Circulating Endothelial Progenitor Cells among Childhood Cancer Survivors Treated with and without Radiation}

Keywords: Pediatric cancers; Endothelial cell functions; Long-tem toxicities; Radiation induced late effects; Childhood cancer survivors

\begin{abstract}
Radiation during childhood cancer treatment increases the propensity to atherosclerotic cardiovascular disease among adult survivors of childhood cancer. This is thought to be mediated through the damage to the underlying vascular endothelium. Endothelia progenitor cells (EPCs) involved in vascular end othelial repair after its damage may be affected by radiation therapy but have never been investigated in adult survivors of childhood cancer. In this pilot study, utilizing multi-parametric flow cytometry, endothelial colony forming cells (ECFCs), which are the bonafide EPCs, and circulating endothelia cells (CECS), which are not EPCs, were compared between adult survivors of childhood cancer with or without radiation exposure. In addition, their associations with blood-pressure, physical activity and diet were examined. Survivors who received radiotherapy had lower ECFCs and CECs $(\mathrm{p}<0.05)$ compared to those without it. Significant positive correlations included physical activity with ECFCs and diet with CECs, while blood-pressure negatively correlated with ECFCs. Further evaluation is needed to examine the effect of radiation and modifiable risk factors on ECFCs and CECs. The preliminary findings from this study suggest evidence of the role of ECFCs as biomarkers of vascular injury following treatment for childhood cancer that may help in early identification of survivors at risk for cardiovascular disease.
\end{abstract}

\section{Introduction}

Although considerable progress has been made in the treatment of childhood cancers, survivors experience significant late-effects such as atherosclerotic cardiovascular disease at a relatively higher rate compared to non-cancer sibling controls [1-3]. The pathogenesis of atherosclerotic cardiovascular disease is rooted in the vascular endothelial damage brought about by radiation and chemotherapy drugs like anthracyclines and cisplatin. The vascular endothelial damage is accelerated with cardiovascular risk factors such as hypertension or lifestyles such as physical inactivity [4-6]. In vitro studies have shown that radiation therapy causes the endothelial progenitor cells of the vascular endothelium, called endothelial colony forming cells (ECFCs), to undergo large-scale senescence, a forerunner of vascular damage and subsequent atherosclerosis [7]. Circulating ECFCs have robust proliferative potential and form perfused new blood vessels in vivo, thereby playing an important role in the repair of damaged vascular endothelium [8,9]. Utilizing a novel multi-parametric flow-cytometry (MPFC) protocol, our group has phenotypically defined the ECFCs by the expression of CD $31^{+}$,
Journal of

Hematology \& Thrombosis

\begin{abstract}
Kamnesh Pradhan ${ }^{1-3 *}$, Julie Mund ${ }^{2-4}$, Jamie Case ${ }^{2-5}$, Samir Gupta ${ }^{6}$, Ziyue Liư , Wambui GathiruaMwangi ${ }^{8}$, Anna McDaniel ${ }^{9}$, Jamie Renbarger ${ }^{1-3}$ and Victoria Champion ${ }^{10}$
\end{abstract}

${ }^{1}$ Department of Pediatric Hematology-Oncology, Riley Hospital for Children, Indianapolis, Indiana, USA

${ }^{2}$ Melvin and Bren Simon Cancer Center, Indiana University Melvin, Indiana, USA

${ }^{3}$ Department of Pediatrics, Scripps Clinic Medical Group, Center for Organ and Cell Transplantation, La Jolla, California, USA

${ }^{4}$ Herman B Wells Center for Pediatric Research, Indiana University School of Medicine, Indianapolis, Indiana, USA

${ }^{5}$ Scripps Clinic Medical Group, Center for Organ and Cell Transplantation, La Jolla, California, USA

${ }^{6}$ Division of Infectious Diseases, Department of Medicine, Indiana University School of Medicine, Indianapolis, Indiana, USA

${ }^{7}$ Division of Biostatistics, Indiana University School of Medicine, Indianapolis, USA

${ }^{8}$ Fairbanks School of Public Health, Indianapolis, Indiana, USA

${ }^{9}$ University of Florida School of Nursing, Gainesville, Florida, USA

${ }^{10}$ Indiana University School of Nursing, Indianapolis, Indiana, USA

*Address for Correspondence:

Kamnesh Pradhan, Department of Pediatric Hematology-Oncology, Riley Hospital for Children, Indianapolis, 705 Riley Hospital Dr, RI-4340, Indianapolis, IN 46202, USA, Tel: (317)-944-8784; Fax: (317)-948-0616; E-Mail: pkamnesh@iu.edu

Submission: 19 June, 2015

Accepted: 31 July, 2015

Published: 05 August, 2015

Copyright: (c) 2015 Pradhan K, et al. This is an open access article distributed under the Creative Commons Attribution License, which permits unrestricted use, distribution, and reproduction in any medium, provided the original work is properly cited.

CD34 $4^{\text {bright }}$, CD45, $\mathrm{AC} 133, \mathrm{CD} 14, \mathrm{CD} 41 \mathrm{a}, \mathrm{CD} 235 \mathrm{a}^{-}$and LIVE/DEAD Violet antigens $[10,11]$. Our group then phenotypically sorted these cells and validated their endothelial characteristics by outgrowth cell culture and expanded endothelial phenotyping, they have colony forming and proliferative potential and formed new blood vessels in vivo [11]. Endothelial cells that express CD31, CD45, CD34 dim and $\mathrm{AC} 133$ antigens are mature, apoptotic cells that cannot form in vivo blood vessels, are sloughed off from the vessel wall and are termed circulating endothelial cells (CECs) $[10,11]$. To verify the apoptotic nature of these cells, our group magnetically isolated these cells and further characterized them through further endothelial cell phenotyping, cell culture where they were unable to form colonies, and apoptotic stains [11].

In contrast to radiation, lifestyle factors such as physical activity has a very favorable effect on endothelial structure and function through its effect on endothelial progenitor cells as seen in the adult non-cancer population $[12,13]$. Therefore, we decided to examine the differences among ECFCs and CECs in survivors with and without radiation exposure and their association with cardiovascular risk factors such as blood-pressure and lifestyle factors such as physical activity and diet. 
Citation: Pradhan K, Mund J, Case J, Gupta S, Liu Z, et al. Differences in Circulating Endothelial Progenitor Cells among Childhood Cancer Survivors Treated with and without Radiation. J Hematol Thromb 2015;1(1): 4.

\section{Materials and Methods}

A pilot study was planned to recruit 2 cohorts consisting of young adult survivors (current age $\geq 18$ years but $<30$ years) who had received chemotherapy but differed by their exposure to radiotherapy. The survivors were recruited over a 12 month period from a large, university-based, tertiary children's outpatient oncology clinic located in the Midwestern United States. Study protocol was approved by the institutional review board.

Self-reported physical activity and diet were obtained from a questionnaire adapted from the Behavioral Risk Factor Surveillance System (BRFSS) survey questions [14,15]. A higher score implied higher self-reported physical activity and a diet higher in fruits and vegetables.

\section{Mononuclear cell isolation, flow cytometry acquisition and analysis}

A 7-color MPFC assay was conducted based on previously published studies by our group [16,17]. Briefly, mononuclear cells isolated with ficoll density gradient centrifugation were stained with antibodies against cell surface antigens, CD34, AC133, CD31, CD45, CD14, and CD16 as well as a viability marker (LIVE/DEAD) and glycophorin A, for the exclusion of dead cells and red blood cells respectively. Fluorescence minus one (FMO) controls were used as positive gating controls are shown in Figure 1. The frequency of phenotypically defined cell populations were acquired using an LSR II flow cytometer equipped with $405 \mathrm{~nm}$ violet, $488 \mathrm{~nm}$ blue, and 633 $\mathrm{nm}$ red laser. All samples were run uncompensated and analyzed using FlowJo 9.7.4 software (Tree Star Inc.) as previously described. The CECs and ECFCs were expressed as the percentage of the total mononuclear cell population.

\section{Statistical analyses}

Categorical variables were compared between groups using the Fisher's exact test. For comparing continuous variables, Student's t-test was used and for correlations among continuous variables, Pearson's correlations were tested unless normality assumptions were violated, in which case Wilcoxon's rank sum tests were used for

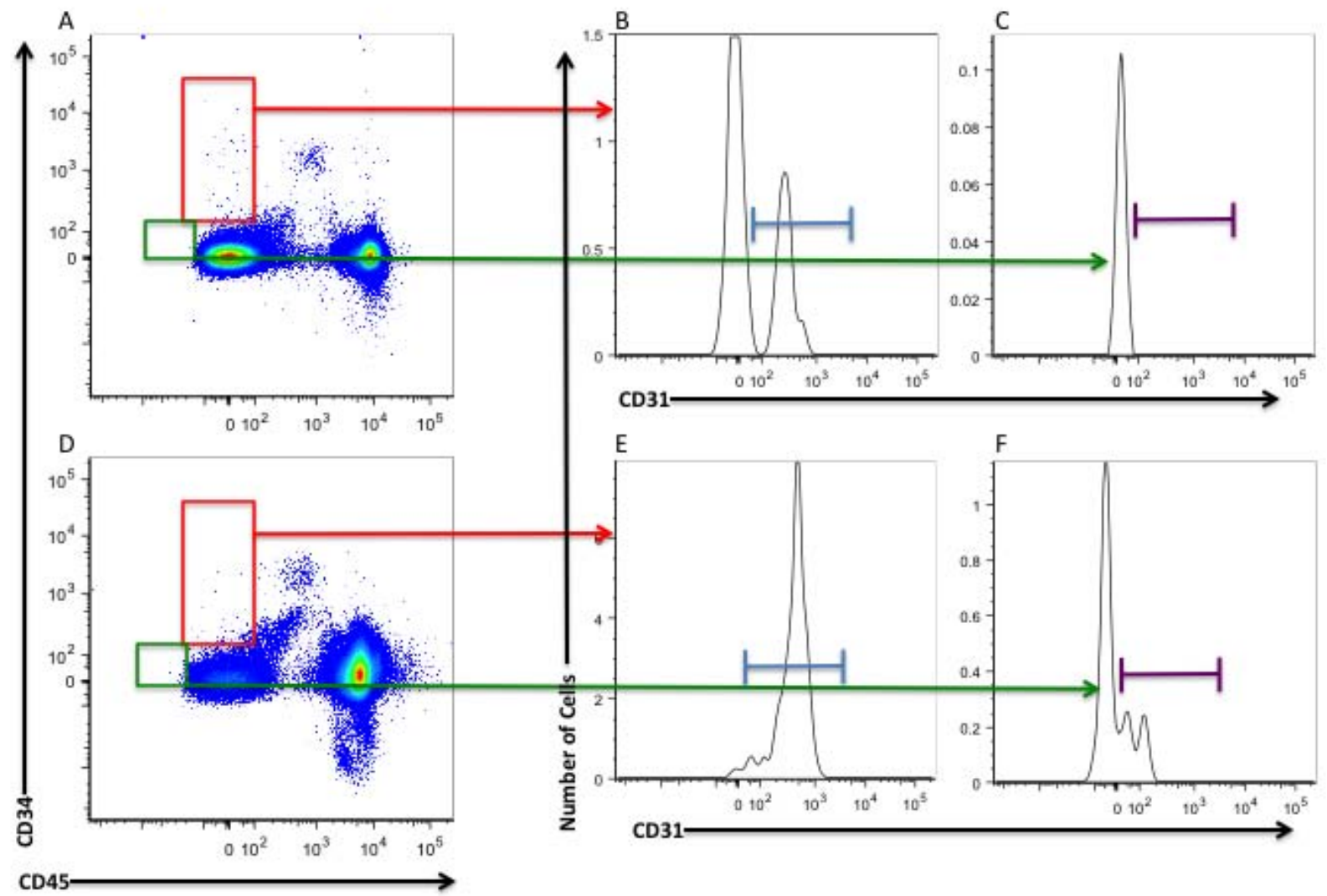

Figure 1: Representative gating strategy of ECFC and CEC populations using Flow Cytometry. Following exclusion of dead cells, low side scatter mononuclea cells were plotted to show CD34 and CD45 (Radiation (XRT): A, Non-XRT: D). CD45 CD34 ${ }^{+}$cells were gated [red] and subsequently gated for CD31 to verify endothelial phenotype (XRT: B; Non-XRT: E). CD45 CD34 dim cells were gated [green] and subsequently gated for CD31 to verify endothelial phenotype (XRT: C; Non-XRT: F). 
Citation: Pradhan K, Mund J, Case J, Gupta S, Liu Z, et al. Differences in Circulating Endothelial Progenitor Cells among Childhood Cancer Survivors Treated with and without Radiation. J Hematol Thromb 2015;1(1): 4.

comparisons and Spearman's rank based for correlations. All analyses were performed using the SAS version 9.3 (SAS Institute, Cary, North Carolina).

\section{Results}

Twenty-four childhood cancer survivors were recruited over 12 months but were not evenly distributed based on radiation exposure. There were no differences between the 2 groups based on age and gender. Of the 24 survivors, 15 received chemotherapy with radiation. Detailed radiation fields and doses are shown in Table 1. Eight survivors with acute leukemia received the same chemotherapy as those with radiation exposure but without any radiotherapy. Majority of the survivors $(n=17)$ had a history of acute leukemia while the rest had solid tumors (6 with brain tumors and 1 with Ewing's sarcoma). The average years from end of treatment to study visit was 8.5 years $(S D=3)$. Eighteen out of 24 survivors received anthracyclines, of which 9 received anthracyclines with radiation. Six of the 24 survivors received cisplatin with radiation without any anthracyclines. The mean percentages of ECFCs for the radiated cohort was $0.0231(\mathrm{SD}=0.036)$ and for the non-radiated cohort was 0.0561 ( $\mathrm{SD}=0.048$ ). The mean percentages of CECs for the radiated cohort was $0.000837(\mathrm{SD}=0.0022)$ and for the non-radiated cohort was $0.00129(\mathrm{SD}=0.0012)$. Those who received radiation had significantly lower levels of ECFCs and CECs ( $p=0.03$ and 0.02 respectively) compared to those who received chemotherapy without radiation (as shown in Figure 2). Among all survivors, there was a significant negative correlation between systolic blood pressure and ECFCs $(\mathrm{p}=0.015$, spearman $\mathrm{rho}=0.48)$. In addition, among all survivors, there was a significant positive correlation between selfreported physical activity and ECFCs $(\mathrm{p}=0.0339$, spearman $\mathrm{rho}=0.43)$ and diet and CECs $(\mathrm{p}=0.02$, spearman $\mathrm{rho}=0.45)$.

\section{Discussion}

This is the first study showing an inhibitory effect of radiotherapy on both the mature (CECs) and progenitor endothelial cell (ECFC) populations among survivors late into the survivorship period (as shown in Table 2). This significant decline of both endothelial cell populations in the radiated cohort confirms the pre-clinical studies showing sensitivity of the ECFCs to radiation greater than 10 GY leading to large-scale radiation induced senescence a cellular phenotype linked to the premature development of atherosclerosis and vasculopathies $[17,18]$. However, the pre-clinical studies were carried out immediately after radiation therapy in contrast to our survivor population where a significant decline among endothelial cells was seen many years following it (median: $9.6 \pm 3 \mathrm{yrs}$ ). This long-term effect may indicate lesser capacity for the ECFCs to repair,

Table 1: Treatment groups of all survivors.

\begin{tabular}{|l|c|c|c|}
\hline \multicolumn{1}{|c|}{ Treatment Groups } & $\begin{array}{c}\text { Acute Leukemia } \\
(\mathbf{n = 1 7})\end{array}$ & $\begin{array}{c}\text { Solid Tumors } \\
(\mathbf{n = 7})\end{array}$ & Total \\
\hline With XRT & 8 & 7 & 15 \\
\hline Without XRT & 9 & -- & 9 \\
\hline Cisplatin & -- & -- & 0 \\
\hline Anthracyclines & 9 & 0 & 9 \\
\hline Cisplatin + XRT & -- & 6 & 6 \\
\hline Anthracyclines + XRT & 8 & 1 & 9 \\
\hline SCT & 3 & -- & 3 \\
\hline
\end{tabular}

XRT: Radiation Therapy; SCT: Stem Cell Transplantation

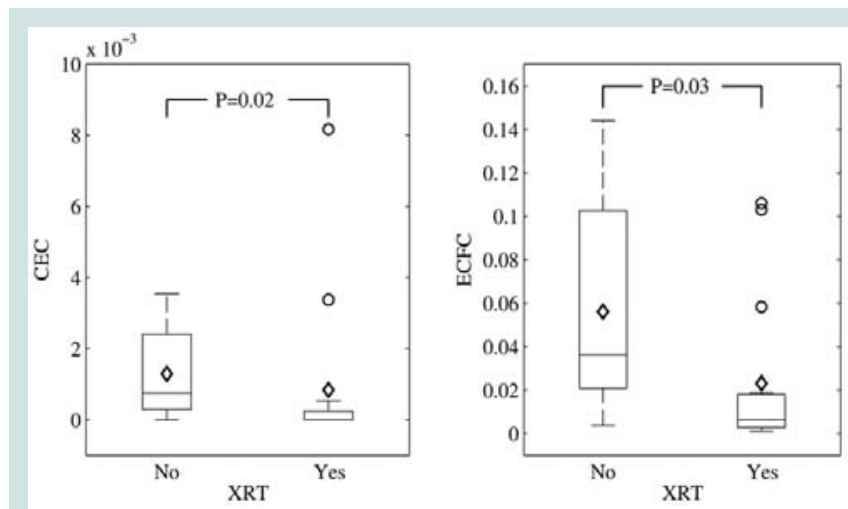

Figure 2: Box-plots showing levels of circulating endothelial cells (CECs) and endothelial colony forming cells (ECFCs) among childhood cancer survivors treated with and without Radiation (XRT).

Table 2: Clinical data of the survivors who received Radiotherapy.

\begin{tabular}{|c|c|c|c|}
\hline Primary diagnosis & Field of XRT & $\begin{array}{l}\text { XRT dose } \\
\text { (Gy) }\end{array}$ & $\begin{array}{c}\text { Time-Interval } \\
\text { between XRT and } \\
\text { Study Visit (years) }\end{array}$ \\
\hline $\begin{array}{l}\text { B-ALL with } \\
\text { testicular relapse }\end{array}$ & Bilateral Testicles & 21 & 8 \\
\hline B-ALL with CNS+ & Cranial & 18 & 16 \\
\hline B-ALL relapse & CSI + Cranial boost & $18+6$ & 10 \\
\hline APML with CNS+ & CSI & 21.6 & 5 \\
\hline B-ALL with relapse & $\begin{array}{l}\text { CSI + Cranial boost } \\
+\mathrm{TBI}\end{array}$ & $18+6+13.5$ & 10 \\
\hline $\mathrm{Ph}+\mathrm{ALL}$ & TBI + Cranial boost & $12+6$ & 7 \\
\hline B-ALL with CNS+ & Cranial & 18 & 10 \\
\hline B-ALL with CNS+ & CSI & $18+6$ & 13 \\
\hline Medulloblastoma & CSI + PF boost & $23.4+55.4$ & 8 \\
\hline Medulloblastoma & CSI + PF boost & $23.4+55.4$ & 9 \\
\hline CNS/PNET & $\begin{array}{l}\mathrm{CSI}+\text { tumor bed } \\
\text { boost }\end{array}$ & $36+50.4$ & 6 \\
\hline Medulloblastoma & CSI + PF boost & $36+50.5$ & 7 \\
\hline Pineoblastoma & $\begin{array}{l}\mathrm{CSI}+\text { tumor bed } \\
\text { boost }\end{array}$ & $36+50.4$ & 7 \\
\hline Medulloblastoma & CSI + PF boost & $23.4+55.4$ & 13 \\
\hline Ewing's Sarcoma & $\begin{array}{l}\text { Focal (Left Parietal } \\
\text { Skull) }\end{array}$ & $45-54$ & 16 \\
\hline
\end{tabular}

XRT: Radiation Therapy; B-ALL: Precursor-B Acute Lymphoblastic Leukemia; CNS+: Central Nervous System is positive for leukemic infiltration; APML: Acute Promyelocytic Leukemia; Ph+ ALL: Philadelphia chromosome ALL; CNS/PNET: Peripheral Neuro-ectodermal Tumor of the Brain; CSI: Cranio-Spinal; boost: Additional XRT; PF: Posterior fossa of the Brain; TBI: Total Body Irradiation; Focal: tumor and surrounding margin only; Gy: Gray (unit of radiation dose)

remodel, and form new perfused blood vessels. The persistent vessel wall damage and a lower capacity to form collateral vessels accelerate atherosclerosis. This may also explain the significant decline in CECs, which are mature, apoptotic cells that are typically sloughed off from the vessels during vessel remodeling.

The significant negative association of systolic blood pressure with ECFCs greatly underscores the importance of altering modifiable risk factors such as hypertension that can adversely affect vascular health and predispose to atherosclerotic cardiovascular disease [19]. The 
Citation: Pradhan K, Mund J, Case J, Gupta S, Liu Z, et al. Differences in Circulating Endothelial Progenitor Cells among Childhood Cancer Survivors Treated with and without Radiation. J Hematol Thromb 2015;1(1): 4.

strong positive correlation of physical activity with ECFCs should be encouraging to survivors and is consistent with recent findings where physical activity enhanced endothelial progenitor cell and endothelial function in the non-cancer population [12]. The ECFCs may mediate the role of physical activity in maintaining robust cardiovascular health. Therefore, physical activity among cancer survivors could potentially alleviate the detrimental effects of radiation on the vascular endothelium. The reason for the significant association between CECs and a diet higher in fruits and vegetables as seen in our study is not known and is being further investigated at this time.

The limitations of this study are the small sample size, crosssectional study design and use of self-report to assess physical activity and diet measures. However, despite these limitations we found statistically significant relationships underscoring the probability that our findings can be duplicated. Although we had information of the fields and doses of radiation, being a pilot study, we did not have the ability to look at the relationship between these radiation parameters and ECFCs and CECs. In addition, contemporary pediatric cancer therapies are complex involving a combination of multiple different chemotherapy regimens with or without radiation. Evaluating and comparing chemotherapy regimens among the 2 cohorts and then adjusting for the different treatment effects was beyond the scope of this pilot study. So we examined differences in regard to radiation exposure that is the strongest causation of vascular dysfunction. A larger study adjusting for other treatment effects is warranted.

In conclusion, the novel findings from this pilot study show the negative effect of radiation exposure on endothelial progenitor cells such as ECFCs. The role of the ECFCs as a potential biomarker to identify childhood cancer survivors at risk for atherosclerotic cardiovascular disease and the effect of lifestyle changes such as physical activity on this cellular population will need to be confirmed in a larger, longitudinal study.

\section{References}

1. Bowers DC, Liu Y, Leisenring W, McNeil E, Stovall M, et al. (2006) Lateoccurring stroke among long-term survivors of childhood leukemia and brain tumors: a report from the childhood cancer survivor study. J Clin Oncol 24: 5277-5282.

2. Oeffinger KC, Mertens AC, Sklar CA, Kawashima T, Hudson MM, et al. (2006) Chronic health conditions in adult survivors of childhood cancer. N Engl J Med 355: 1572-1582.

3. Mertens AC, Liu Q, Neglia JP, Wasilewski K, Leisenring W, et al. (2008) Cause-specific late mortality among 5-year survivors of childhood cancer: the childhood cancer survivor study. J Natl Cancer Inst 100: 1368-1379.

4. Brouwer CA, Postma A, Hooimeijer HL, Smit AJ, Vonk JM, et al. (2013) Endothelial damage in long-term survivors of childhood cancer. J Clin Oncol 31: 3906-3913

5. Chow AY, Chin C, Dahl G, Rosenthal DN (2006) Anthracyclines cause endothelial injury in pediatric cancer patients: a pilot study. J Clin Oncol 24: 925-928.

6. Dillenburg RF, Nathan P, Mertens L (2013) Educational paper: decreasing the burden of cardiovascular disease in childhood cancer survivors: an update for the pediatrician. Eur J Pediatr 172: 1149-1160.

7. Mendonca MS, Chin-Sinex H, Dhaemers R, Mead LE, Yoder MC, et al. (2011) Differential mechanisms of $x$-ray-induced cell death in human endothelia progenitor cells isolated from cord blood and adults. Radiat Res 176: 208

8. Ingram DA, Mead LE, Moore DB, Woodard W, Fenoglio A, et al. (2005)
Vessel wall-derived endothelial cells rapidly proliferate because they contain a complete hierarchy of endothelial progenitor cells. Blood 105: 2783-2786.

9. Yoder MC, Mead LE, Prater D, Krier TR, Mroueh KN, et al. (2007) Redefining endothelial progenitor cells via clonal analysis and hematopoietic stem/ progenitor cell principals. Blood 109: 1801-1809.

10. Estes ML, Mund JA, Ingram DA, Case J (2010) Identification of endothelial cells and progenitor cell subsets in human peripheral blood. Curr Protoc Cytom Chapter 9: Unit 9.33.1-11.

11. Mund JA, Estes ML, Yoder MC, Ingram DA Jr, Case J (2012) Flow cytometric identification and functional characterization of immature and mature circulating endothelial cells. Arterioscler Thromb Vasc Biol 32: 1045-1053.

12. De Biase C, De Rosa R, Luciano R, De Luca S, Capuano E, et al. (2014) Effects of physical activity on endothelial progenitor cells (EPCs). Front Physiol 4: 414.

13. Ribeiro F, Ribeiro IP, Alves AJ, do Céu Monteiro M, Oliveira NL, et al. (2013) Effects of exercise training on endothelial progenitor cells in cardiovascular disease: a systematic review. Am J Phys Med Rehabil 92: 1020-1030.

14. Holtzman D (2003) The behavioral risk factor surveillance system. In Blumenthal DS, DiClemente RJ (Eds.), Community-based health research: Issues and methods. Springer Publishing Company, New York, pp. 115-131.

15. CDC (2011) Youth risk behavior surveillance system (YRBSS). Centers for Disease Control and Prevention.

16. Estes ML, Mund JA, Mead LE, Prater DN, Cai S, et al. (2010) Application of polychromatic flow cytometry to identify novel subsets of circulating cells with angiogenic potential. Cytometry A 77: 831-839.

17. Pradhan KR, Mund JA, Johnson C, Vik TA, Ingram DA, et al. (2011) Polychromatic flow cytometry identifies novel subsets of circulating cells with angiogenic potential in pediatric solid tumors. Cytometry B Clin Cytom 80: 335-338

18. Ingram DA, Krier TR, Mead LE, McGuire C, Prater DN, et al. (2007) Clonogenic endothelial progenitor cells are sensitive to oxidative stress. Stem Cells 25: 297-304.

19. Pirro M, Schillaci G, Menecali C, Bagaglia F, Paltriccia R, et al. (2007) Reduced number of circulating endothelial progenitors and HOXA9 expression in CD34+ cells of hypertensive patients. J Hypertens 25: 20932099.

\section{Acknowledgements}

We thank Emily Sims and Mathew Repass of the Angio Bio Core at the Indiana University Simon Cancer Center (IUSCC) for processing the peripheral blood samples for this study. We also acknowledge the assistance and state-of the art facilities of the Flow Cytometry Resource Facility at the IUSCC. We would also like to thank Leah Engelstad and Shannon Aranjo from Pediatric Clinical Research in helping to recruit the research subjects and complete all research related procedures.

\section{Financial support}

Supported by the Indiana University Health Values Funds (VFR-342, K.R.P), and by a Project Development Team within the Indiana CTSI (NIH/ NCRR Grant Number TR000006, K.R.P). K.R.P is a post-doctoral fellow and W.G-M. a pre-doctoral fellow supported by the Training in Research for Behavioral Oncology and Cancer Control Program funded by the National Cancer Institute of the National Institutes of Health (Award Number R25CA117865). The content is solely the responsibility of the authors and does not necessarily represent the official views of the $\mathrm{NIH}$. 